\title{
Towards a New $\mu \rightarrow$ e $\gamma$ Search with the MEG II Experiment: From Design to Commissioning
}

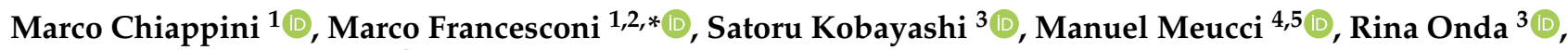 \\ Patrick Schwendimann ${ }^{6,7}$ (D) and on behalf of the MEG II Collaboration ${ }^{\dagger}$
}

check for

updates

Citation: Chiappini, M.; Francesconi M.; Kobayashi, S.; Meucci, M.; Onda,

R.; Schwendimann, P.; on behalf of the MEG II Collaboration. Towards a New $\mu \rightarrow$ e $\gamma$ Search with the MEG II Experiment: From Design to Commissioning. Universe 2021, 7, 466 https://doi.org/10.3390/

universe7120466

Academic Editors: Robert H. Bernstein and Bertrand Echenard

Received: 12 October 2021

Accepted: 26 November 2021

Published: 30 November 2021

Publisher's Note: MDPI stays neutral with regard to jurisdictional claims in published maps and institutional affiliations.

Copyright: (c) 2021 by the authors. Licensee MDPI, Basel, Switzerland. This article is an open access article distributed under the terms and conditions of the Creative Commons Attribution (CC BY) license (https:/ / creativecommons.org/licenses/by/ $4.0 /)$.
1 INFN Sezione di Pisa, Largo B. Pontecorvo 3, 56127 Pisa, Italy; marco.chiappini@pi.infn.it

2 Dipartimento di Fisica, dell'Università, Largo B. Pontecorvo 3, 56127 Pisa, Italy

3 ICEPP, The University of Tokyo, 7-3-1 Hongo, Bunkyo-ku, Tokyo 113-0033, Japan; satoruk@icepp.s.u-tokyo.ac.jp (S.K.); onda@icepp.s.u-tokyo.ac.jp (R.O.)

4 INFN Sezione di Roma, Piazzale A. Moro 2, 00185 Rome, Italy; manuel.meucci@roma1.infn.it

5 Dipartimento di Fisica, dell'Università "Sapienza", Piazzale A. Moro 2, 00185 Rome, Italy

6 Paul Scherrer Institut PSI, 5232 Villigen, Switzerland; patrick.schwendimann@psi.ch

7 Swiss Federal Institute of Technology ETH, 8093 Zurich, Switzerland

* Correspondence: marco.francesconi@pi.infn.it; Tel.: +39-050-2214-304

+ MEG II Collaboration are listed in acknowledgments.

Abstract: The MEG experiment represents the state of the art in the search for the Charged Lepton Flavour Violating $\mu^{+} \rightarrow \mathrm{e}^{+} \gamma$ decay. With its first phase of operations at the Paul Scherrer Institut (PSI), MEG set the most stringent upper limit on the $\mathrm{BR}\left(\mu^{+} \rightarrow \mathrm{e}^{+} \gamma\right) \leq 4.2 \times 10^{-13}$ at $90 \%$ confidence level, imposing one of the tightest constraints on models predicting LFV-enhancements through new physics beyond the Standard Model. An upgrade of the MEG experiment, MEG II, was designed and it is presently in the commissioning phase, aiming at a sensitivity level of $6 \times 10^{-14}$. The MEG II experiment relies on a series of upgrades, which include an improvement of the photon detector resolutions, brand new detectors on the positron side with better acceptance, efficiency and performances and new and optimized trigger and DAQ electronics to exploit a muon beam intensity twice as high as that of MEG $\left(7 \times 10^{7} \mu^{+} / \mathrm{s}\right)$. This paper presents a complete overview of the MEG II experimental apparatus and the current status of the detector commissioning in view of the physics data taking in the upcoming three years.

Keywords: Lepton Flavour Violation; muons; rare decays; intensity frontier experiment; physics beyond Standard Model; particle detector

\section{Introduction}

For more than half a century, the search for Charged Lepton Flavour Violation (CLFV) processes provided important clues towards our current understanding of the Standard Model (SM) of particle physics [1]. Starting as early as 1947, the first upper limit of the muon decay into an electron and a photon (what we call $\mu \rightarrow$ e $\gamma$ today) was established by Hincks and Pontecorvo [2]; it opened the way for the introduction of the neutrino and later of lepton flavour as conserved quantity.

In the last decades, the observation of neutrino oscillations [3] exposed the empirical and approximate nature of the lepton flavour symmetry, but they produce extremely small Branching Ratios (BR) for their charged counterparts in the SM $\left(\ll 10^{-50}\right)$, way beyond experimental sensitivities $[4,5]$. On the other hand, any theory trying to explain the flavour structure of the SM [6] (including the recent measurement of the muon g-2 [7]) has to now cope with very stringent experimental limits from the charged lepton sector. At the same time, an observation of a CLFV process will be immediate evidence of new pyhsics beyond the SM.

The $\mu^{+} \rightarrow \mathrm{e}^{+} \gamma$ process is still very sensitive to new physics, with a current upper limit on the BR of $4.2 \times 10^{-13}$ at $90 \%$ confidence level set by the MEG experiment [8] at 
the Paul Scherrer Institut (PSI). It is also considered highly complementary to other muon CLFV processes, in particular the $\mu \rightarrow 3 \mathrm{e}$ and $\mu^{-} \rightarrow \mathrm{e}^{-}$conversion in atomic nuclei [9]. In particular, the $\mu^{+} \rightarrow \mathrm{e}^{+} \gamma$ search with the MEG experiment upgrade, MEG II, in terms of new physics reach, is competitive with the new generation of CLFV experiments such as Mu3e [10], Mu2e [11], COMET [12] and DeeMe [13].

\section{Materials and Methods}

The MEG II experiment builds on the knowledge obtained by operating the former MEG experiment [14]. The detection strategy remains the same: a beam of positive muons is stopped on a thin plastic target where they decay at rest to exploit the two body kinematics of the $\mu^{+} \rightarrow \mathrm{e}^{+} \gamma$ process. The target is located at the center of a magnetic spectrometer used to track the candidate positron. At the same time, a photon detector measures the timing, energy and the conversion position of the photon, completing the experiment observables.

There are two sources of background in the MEG apparatus [4]: the first is the irreducible physics background originating from the Radiative Muon Decay (RMD, $\mu^{+} \rightarrow \mathrm{e}^{+} v \bar{v} \gamma$ ), when the neutrinos carry a small fraction of the available energy. This process was measured in MEG [15] and can be suppressed by a very precise (at the 1\% level) detection of the photon energy spectrum at the kinematic endpoint. .qThe second one, the accidental background, constitutes the dominant source of background for the MEG experiment. It arises from the very high muon stop rate which enhances the probability of a time coincidence of a positron from the dominating "Michel" muon decay $\left(\mu^{+} \rightarrow \mathrm{e}^{+} v \bar{v}\right)$ with photons from any other source. Being an accidental coincidence, this background can be mitigated by measuring the difference in particle emission time with very high accuracy (at the 100 ps level). The suppression of both backgrounds is enhanced by an angular resolution on the order of a few mrad.

While Michel positrons are abundant in the spectrometer volume due to the physics of this muon decay, the precise measurement of the $\gamma$-ray spectrum close to the signal energy reduces the accidental background. The spectrum is in fact dominated by the aforementioned RMD decays, with a non-negligible contribution (35\% of all events with $E_{\gamma}>48 \mathrm{MeV}$ ) coming from the annihilation in flight (AIF, $\mathrm{e}^{+} \mathrm{e}^{-} \rightarrow \gamma \gamma$ ) of a positron from Michel decay inside the spectrometer. Therefore using detectors with small interaction length and identifying high energy photons from RMD events is a key ingredient for the MEG II apparatus.

With the detectors shown in Figure 1, MEG II aims at an order of magnitude improvement [16] in the sensitivity with respect to MEG by increasing all resolutions by roughly a factor 2 with respect to the former experiment. This required a major upgrade of the existing Liquid Xenon (LXe) detector as well as a complete redesign of the positron spectrometer consisting of the Cylindrical Drift CHamber $(\mathrm{CDCH})$ and the pixelated Timing Counter (pTC). The Radiative Decay Counter (RDC) was added to tag and remove RMD events that otherwise would contribute to the accidental background. The target deformation systematic effect, that was a significant limitation in the former experiment, will be addressed by imaging markers printed on the target with cameras.

In addition to providing increased resolutions, the new apparatus has to sustain a higher rate of muons (up to $7 \times 10^{7} \mu^{+} / \mathrm{s}$ ), so to collect the required statistics within a reasonable time frame. Both requirements pushed towards a finer segmentation with a correspondingly increased complexity of the detectors as well as of the trigger and data acquisition system. 


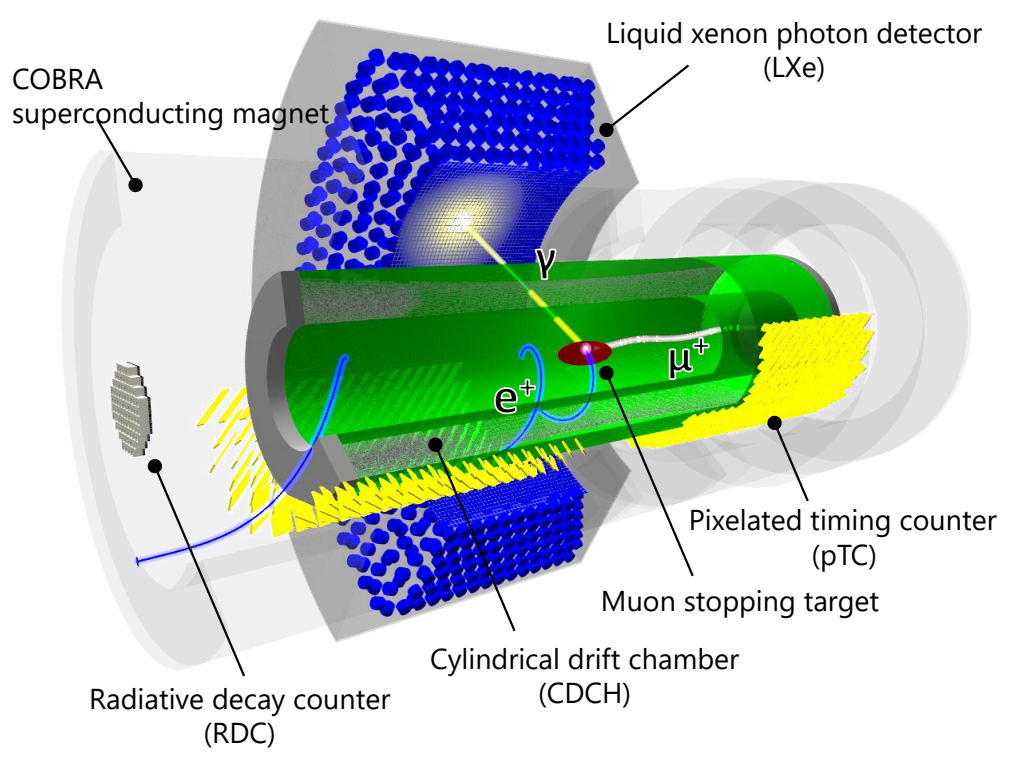

Figure 1. Drawing of the detectors constituting the MEG II apparatus.

\subsection{Beamline and Target}

The MEG II experiment makes use of the PiE5 secondary beamline at the Paul Scherrer Institut. This beamline can deliver more than $1 \times 10^{8} \mu^{+} / \mathrm{s}$ in a continuous beam as well as pions and positrons. This continuity of the beam is crucial for the experiment to keep the accidental background at the lowest possible level while still accumulating statistics at acceptable rate to reach the desired sensitivity within a few years of physics data acquisition. The possibility to deliver positrons and pions is used for calibration purposes.

For MEG II, the PiE5 beamline is tuned to transport surface muons, i.e., muons that originate from pion decays on the surface of the muon production target (PSI proton target E [17]). These muons are partially polarized [18] and have a momentum of about $28 \mathrm{MeV}$. Thus they can easily be stopped on a dedicated target. For tuning and beam characterization, a set of dedicated detectors is deployed $[16,19,20]$. Moreover, the beamline features a separator that allows to select between muons and positrons. Rejecting the positrons is of crucial interest to suppress beam related pile-up in the detectors and especially in the RDC.

As in the previous MEG experiment, the muon beam is stopped in a slanted target. The current target is made of polyvinyltoluene. It has a thickness of $130 \mu \mathrm{m}$ and measures $260 \mathrm{~mm} \times 70 \mathrm{~mm}$ without the carbon fiber support frame. The whole target can be remotely shifted from the upstream "parking" position to the experiment center when in measurement position. The parking position is used for calibration purposes when using alternative targets.

In addition, a dot pattern is drawn on the target, which is used to precisely determine position and potential deformations of the target during the measurements. This pattern is monitored by a set of two high resolution, radiation tolerant and magnetic field insensitive cameras. They are equipped with a LED and installed roughly one meter upstream and $10 \mathrm{~cm}$ off-axis, well out of beam path and detector acceptance. The pictures are analyzed to track the changes of the dot pattern. Based on these changes, the target position is extrapolated with a precision better than $100 \mu \mathrm{m}$ on the beam axis coordinate [21,22]. These parameters are essential to the reconstruction algorithms that identify the muon decay position, and thus identify events where the positron-photon pair is emitted in a back to back geometry.

To verify the reconstruction algorithm, holes are punched into the target, which are clearly visible when plotting the reconstructed decay vertex position. The performance of the algorithm is then extracted from that distribution around the edges of the holes. 


\subsection{Positron Spectrometer}

The MEG II $\mathrm{e}^{+}$spectrometer consists of a low-mass cylindrical drift chamber with high granularity and stereo configuration of the drift cells, followed by a pixelated timing counter, based on scintillator tiles read out by SiPMs, for a precise measurement of the $\mathrm{e}^{+}$momentum vector and time respectively. The spectrometer acceptance is increased by more than a factor of two with respect to MEG. Both detectors are placed inside the COBRA (COnstant Bending RAdius) superconducting magnet [23], featuring a gradient axial field ranging from $1.27 \mathrm{~T}$ (center) to $0.49 \mathrm{~T}$ (endcaps). With this field configuration the trajectory of a $\mathrm{e}^{+}$emerging from the $\mu^{+}$stopping target has an approximately constant bending radius that only slightly depends on the polar emission angle (it depends almost exclusively on the total momentum). Moreover, low-momentum positrons emitted at $\approx 90^{\circ}$ with respect to the axis are rapidly swept away.

\subsubsection{Pixelated Timing Counter-pTC}

The timing of the $\mathrm{e}^{+}$is measured by the pixelated timing counter [24]. While its main goal is to measure the time of the $\mathrm{e}^{+}$with high precision, it can also be used for coarse tracking and momentum measurements. It is used to generate the trigger signal from the $\mathrm{e}^{+}$side, as the $\mathrm{CDCH}$ cannot provide a fast enough signal due to the latency introduced by the drift time. The pTC is composed of two identical modules, each one containing 256 counters. Each counter unit is itself composed of a scintillating tile read out by Silicon PhotoMultipliers (SiPM). The high granularity of this design guarantees a good number of hits, improving the overall time and spatial resolutions.

Figure 2a shows one pTC module inside the COBRA volume. It is a $16 \times 16$ matrix organized as follows: 16 lines of cylindrically arranged counters with a $10.3^{\circ}$ interval, each line staggered by a half counter and containing 16 counters with a $5.5 \mathrm{~cm}$ spacing in the longitudinal direction. The counters are tilted at $45^{\circ}$ to be almost perpendicular to the signal $\mathrm{e}^{+}$trajectory. The longitudinal and angular acceptance fully covers the experiment acceptance which is defined by the Liquid Xenon detector, and is the following: $23.0 \mathrm{~cm}<|z|<116.7 \mathrm{~cm}$ and $-165.8^{\circ}<\phi<5.2^{\circ}$, where $z$ is the beam axis coordinate and $\phi$ is the azimuthal angle.

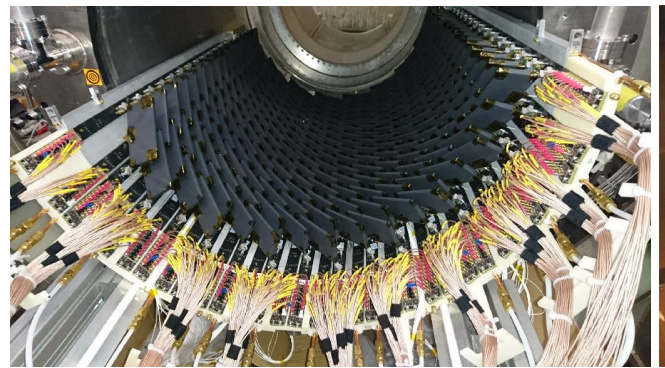

(a)

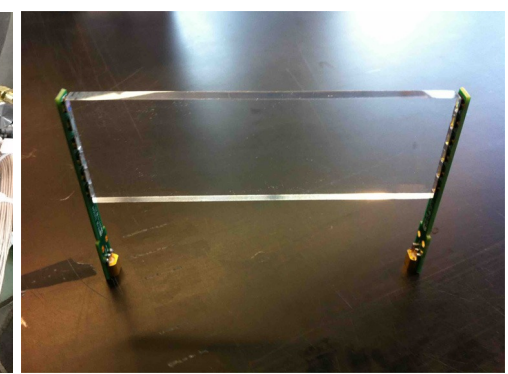

(b)

Figure 2. (a) Picture of a single pTC module installed inside the COBRA volume. At the time of the picture the $\mathrm{CDCH}$ had not been installed yet. (b) Picture of a single pTC tile before the installation inside the detector.

The pTC tiles are made of a plastic scintillator plate coupled to six SiPMs connected in series at each end. The SiPMs are soldered to a PCB and then glued to the scintillator, in order to minimize the material crossed by the $\mathrm{e}^{+}$. There are two types of tiles that differ in the scintillator size $W$, depending on the counter location: $120 \times 40 \times 5 \mathrm{~mm}^{3}$ and $120 \times 50 \times 5 \mathrm{~mm}^{3}$. The difference stems from the dependence of the radial spread of the $\mathrm{e}^{+}$ trajectory on the longitudinal position: the $W=50 \mathrm{~mm}$ counters are placed in the middle longitudinal position, where the radial spread is larger. The scintillator plate is made from BC-422 from Saint Gobain [25] wrapped in a $32 \mu \mathrm{m}$ thick Enhanced Specular Reflector (ESR) [26] film. Furthermore, each counter is wrapped in a $25 \mu \mathrm{m}$ thick black sheet of 
Tedlar before the installation inside the pTC module for extra light tightness. Figure $2 b$ shows a picture of a single tile before the wrapping.

The detector calibration is based on a laser system that distributes a signal to most of the tiles through several optical components [27]. This allows for a precise determination of each counter time offset. The pTC equipment includes also a $1 \mathrm{~kW}$ cooling system $(\sim 350 \mathrm{~W}$ per side are needed to dissipate the heat from the nearby $\mathrm{CDCH}$ front end electronics) operating with water to limit the dark count rate in the SiPMs [28].

\subsubsection{Cylindrical Drift $\mathrm{CHamber-CDCH}$}

The new drift chamber $[29,30]$ of the MEG II experiment is a single volume detector with cylindrical symmetry along the $\mu^{+}$beam. The active volume extends for $1.91 \mathrm{~m}$ in length and $17 \div 29 \mathrm{~cm}$ radially, ensuring full azimuthal coverage around the $\mu^{+}$stopping target. This improves the geometric acceptance for signal $\mathrm{e}^{+}$and allows to use new tracking procedures capable to exploit four times more hits than MEG and match the information reconstructed by the CDCH and pTC for a larger tracking efficiency (65\% vs. $40 \%$ in MEG).

The high granularity is ensured by 9 layers of 192 drift cells. Each layer consists of two criss-crossing field wire planes enclosing a sense wire plane. The wires form an angle with the $\mathrm{CDCH}$ axis that increases from about $6^{\circ}$ to $8^{\circ}$ for increasing radius. This stereo angle has an alternating sign, depending on layer, allowing the experiment to reconstruct the longitudinal hit coordinate. The single drift cell is quasi-square with a $20 \mu \mathrm{m}$ Gold-plated Tungsten sense wire surrounded by $40 / 50 \mu \mathrm{m}$ Silver-plated Aluminum field wires, with a 5:1 field-to-sense wire ratio and a total number of $\approx 12,000$ wires. The cell width increases linearly with the radius and also slightly varies along the axis: $6.7 \div 8.7 \mathrm{~mm}$ at the ends, $5.8 \div 7.5 \mathrm{~mm}$ at the center, due to the stereo geometry.

The $\mathrm{CDCH}$ is the first drift chamber ever designed and built in a modular way [31]. In fact, given the high wire density $\left(12\right.$ wires $\left./ \mathrm{cm}^{2}\right)$, the classical feed-through technique with wires anchored to the endplates is hard to implement. The wires are not strung directly on the chamber but soldered at both ends on the pads of two PCBs, which are then radially stacked by means of PEEK spacers in the twelve $30^{\circ}$-sectors of the endplates. In between these sectors, a radial aluminum pillar provides the required mechanical robustness. Figure 3 shows the fully wired detector.

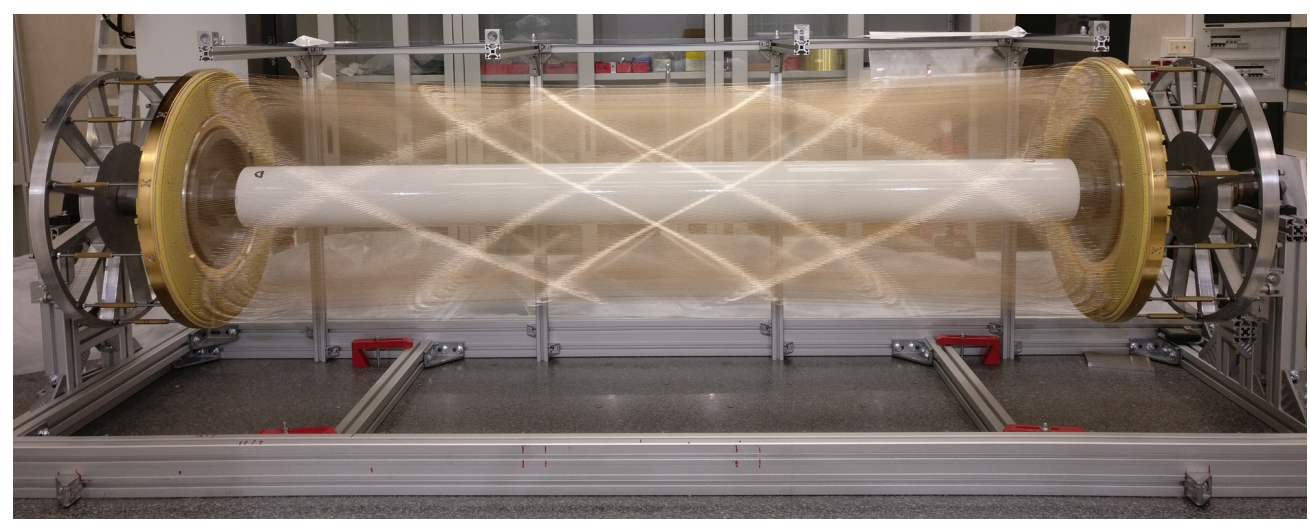

Figure 3. The fully wired MEG II CDCH.

The sensitive volume is filled with a low-mass $\mathrm{He}_{\mathrm{iC}} \mathrm{C}_{4} \mathrm{H}_{10}(90: 10)$ gas mixture [32], with small amounts of additives to improve the operational stability. The gas choice is a good compromise between high transparency and single-hit resolution, that has been measured to be $<120 \mu \mathrm{m}$ [33] (200 $\mu \mathrm{m}$ in MEG) on prototypes. At the innermost radius, a $20 \mu \mathrm{m}$ one-side-Al Mylar foil separates the $\mathrm{CDCH}$ gas volume from the He-filled target region. At the outermost radius, a $2 \mathrm{~mm}$-thick carbon fiber support structure encloses the sensitive volume and keeps the endplates at the correct distance, ensuring the proper mechanical wire tension. All the aforementioned features contribute to minimize the Multiple Coulomb 
Scattering contribution and the total radiation length down to $1.5 \times 10^{-3} \mathrm{X}_{0}$ per track turn $\left(2 \times 10^{-3} \mathrm{X}_{0}\right.$ in MEG).

With the MEG II particle flux ( $>\times 2$ with respect to MEG) the CDCH occupancy per $\mathrm{cm}$ of sense wire is $\approx 23 \mathrm{kHz} / \mathrm{cm}$ at inner radii near the stopping target, decreasing to $<5 \mathrm{kHz} / \mathrm{cm}$ as radius and longitudinal coordinate increase. Ageing tests with prototypes [34] resulted in a gain loss $<20 \%$ per DAQ year for the inner layers. This is an acceptable value that can be recovered by slightly increasing the operating voltage. Fast custom front-end boards [35] supply the HV to the wires from one side and read out the signal at both wire ends. This improves the reconstruction of the longitudinal hit coordinate through the techniques of charge division and time of propagation difference. The active cooling of the electronics is ensured by a chiller system.

Aluminum wire breaking problems arose during the $\mathrm{CDCH}$ assembly and commissioning, despite the fact that all the operations were performed inside cleanrooms with a strict monitoring of the environmental conditions. The problem was carefully investigated using optical inspections with microscopes, chromatography, practical tests and SEM/EDX analyses [36]. A safe procedure to extract the broken wire pieces from the chamber has been developed. Electric field simulations showed that the effect of a missing cathode wire on the $\mathrm{e}^{+}$reconstruction is negligible. Chemical and mechanical analyses showed that the origin of the breaking phenomenon is the chemical corrosion of the $\mathrm{Al}$ core in presence of water condensation of ambient humidity on wires. Keeping the wire volume in dry atmosphere proved effective to stop the development of corrosion.

\subsection{Liquid Xenon Gamma Detector-LXe}

The liquid xenon detector of the MEG II experiment is a C-shape detector responsible for the measurement of energy, time, and conversion point of the photons. It is composed of a $900 \mathrm{~L}$ liquid xenon volume surrounded by photo-sensors. Liquid xenon is cooled down to $165 \mathrm{~K}$ to maintain the liquid state and emits scintillation light in the vacuum ultraviolet (VUV, $\sim 175 \mathrm{~nm}$ ) range.

The MEG liquid xenon detector used 846 photo multiplier tubes (PMTs) to detect scintillation light, but its performance was limited due to the non-uniform coverage of the PMT sensitive area, especially on the photon entrance face. The entrance face of the MEG II detector is instead covered by 4092 Multi-pixel photon counters (MPPC, a family of SiPM) and the other faces are covered by 668 PMTs in total (Figure 4).

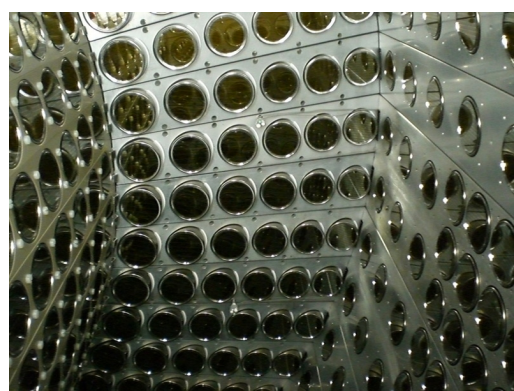

(a)

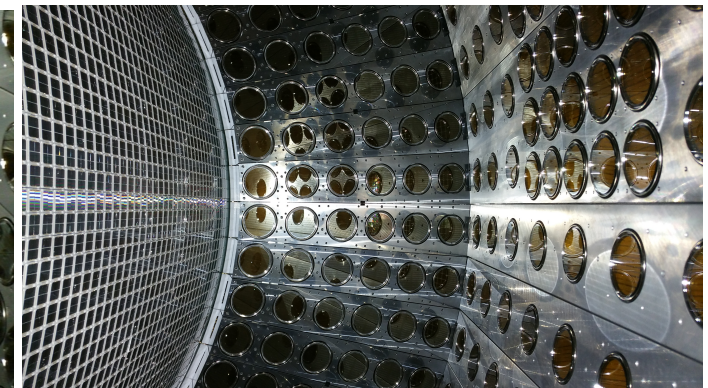

(b)

Figure 4. (a) Picture of photo-sensors mounted on inner faces of the (a) MEG and (b) MEG II liquid xenon detector.

VUV-sensitive MPPCs (VUV-MPPC) were developed by the MEG II experiment in collaboration with Hamamatsu Photonics K.K. [37]. Whereas standard SiPMs are not sensitive to VUV photons due to absorption in surface layers, VUV-MPPC achieve a high Photon Detection Efficiency (PDE) with a reduced absorption in surface layers and an optimized optical matching between layers. VUV-MPPCs with a size of $15 \mathrm{~mm} \times 15 \mathrm{~mm}$ fill the entrance face with small gaps and realize a granular and uniform readout of the entrance face. 
The high granularity and uniformity of the scintillation light readout is a key to precise gamma-ray reconstruction. The resolution of spatially displaced pileup gamma-rays as well as the position and energy resolution for photons converted near the entrance face are expected to be improved with respect to the MEG detector. Furthermore, a relatively low amount of material from the MPPCs contributes to a high detection efficiency (69\%). The expected energy, position, and time core resolutions are $\sim 0.5 \mathrm{MeV}, \sim 2.5 \mathrm{~mm}$, and $\sim 50-70$ ps respectively.

The detector has two intrinsic calibration methods based on LEDs and ${ }^{241}$ Am sources [38]. These sources allow constant monitoring of photo-sensor performances such as gain and PDE. There are also a few external gamma-ray sources for the detector calibration. Two gamma-ray sources are used on a daily basis for the detector monitoring during beam time: $\sim 9 \mathrm{MeV}$ ones from the capture of thermalized neutrons in a Nickel plate and $17.6 \mathrm{MeV}$ ones from a Li target bombarded by a proton beam from a Cockroft-Walton accelerator (CW) [39]. On the other hand, gamma-rays from a neutral pion decay following a charge exchange (CEX) reaction of a negative pion on a proton $\left(\pi^{-}+\mathrm{p} \rightarrow \pi^{0}+n, \pi^{0} \rightarrow \gamma \gamma\right)$ are used for the detector performance evaluation. These two gamma rays have energies ranging from $55 \mathrm{MeV}$ to $83 \mathrm{MeV}$ and exhibit a strong correlation with the corresponding opening angle. This gamma-ray source allows precise measurement of energy and timing resolution close to the signal energy that requires a dedicated setup consisting of $\pi^{-}$beam and $\mathrm{LH}_{2}$ target. For this reason this measurement is performed once per year.

\subsection{Radiative Decay Counter-RDC}

The Radiative Decay Counter was newly installed for the MEG II experiment in order to suppress $\gamma$-ray backgrounds. The RDC aims at identifying $\gamma$-rays from RMD by detecting low energy positrons emitted simultaneously, which are outside the momentum acceptance of the main spectrometer.

RMD events can be identified by considering a time coincidence between the LXe detector and the RDC. However, not only positrons from the RMD but also those from the Michel decay hit the RDC, which can be a background for the RMD identification. Such Michel positrons tend to have higher energies compared to RMD positrons, such that they can be distinguished by measuring their energies.

The RDC is placed on the beam axis since low energy positrons travel along this axis at small radii due to the COBRA magnet. In principle, such a detector can be installed on both the upstream and downstream sides of the target. However, only the downstream RDC is installed until now as the upstream RDC is still under development. The main difficulties are the stringent requirements for its performance while not affecting the high intensity muon beam which passes through it.

The downstream RDC consists of two sub-modules, a timing counter and a calorimeter (Figure 5a). Since Michel positrons hit the RDC at the frequency of $1 \times 10^{7} \mathrm{e}^{+} / \mathrm{s}$, the RDC has a finely segmented structure. The timing counter is composed of 12 fast plastic scintillator bars (BC-418, Saint-Gobain) with $5 \mathrm{~mm}$ thickness. The width of the bars around the center is $1 \mathrm{~cm}$ while it is $2 \mathrm{~cm}$ for the others as the occupancy is higher in the center. The length of the bars ranges from $7 \mathrm{~cm}$ on the outside to $19 \mathrm{~cm}$ in the center. The scintillation light is read out with SiPMs (S13360-3050PE, Hamamatsu Photonics K.K.) attached to the both ends of each scintillator. Two or three SiPMs are connected in series for $1 \mathrm{~cm}$ or $2 \mathrm{~cm}$ wide scintillators, respectively. The calorimeter consists of 76 LYSO crystals (Shanghai Institute of Ceramics) in the shape of $2 \mathrm{~cm}$ side cubes. The scintillation light is read out with one SiPM (S12572-25P, Hamamatsu Photonics K.K.) attached to the back of each crystal. The energy deposit in the calorimeter part provides the additional discrimination power that is needed to separate RMD positrons from the higher energy positrons from Michel decays.

The upstream RDC is in the development phase. As it will be installed in the path of the muon beam, its material thickness has to be small enough not to affect the beam. In addition, it must be finely segmented and have a fast response to distinguish RMD positrons from muons. Moreover, radiation hardness is required to be operational under 
the high intensity beam. A Resistive Plate Chamber (RPC) has been investigated as a candidate (Figure 5b). The RPC is made of parallel resistive plates of positively-charged anodes and negatively-charged cathodes, and a gas mixture for charge multiplication is filled in-between. In order to satisfy the low material budget, a thin polyimide film coated with diamond-like carbon (DLC) is used as the resistive plate [40].

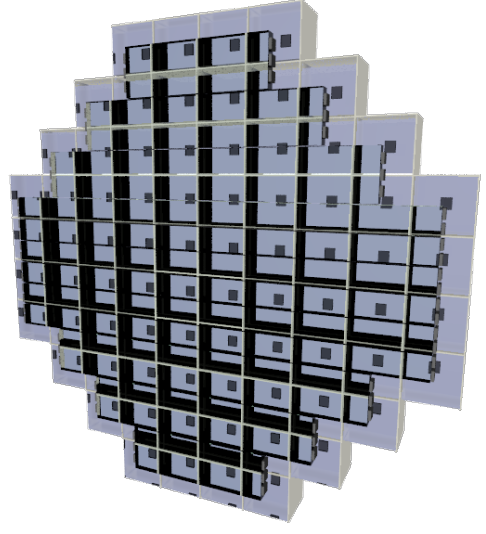

(a)

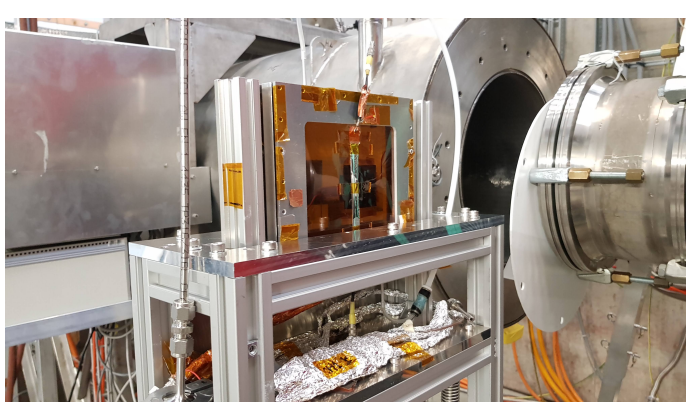

(b)

Figure 5. (a) The downstream RDC. (b) Proto-type RPC tested with the $\mu$ beam.

\subsection{Trigger and Data Acquisition-TDAQ}

The MEG II experiment required a complete redesign of the Trigger and Data Acquisition system (TDAQ) to cope with an almost tripled number of electronic channels. At the same time the new system had to maintain the excellent charge and time resolutions, provided by the DRS4 (Domino Ring Sampler 4) readout ASIC [41] which had already been used in MEG. All the required 8900 channels are successfully in operation in the same rack space used by MEG by developing a new fully integrated TDAQ system, called WaveDAQ [42].

The trigger and data acquisition path were combined in a single readout card called WaveDREAM (Drs4 REAdout Module) which contains, in addition to the DRS4, also a programmable discriminator and an $80 \mathrm{MHz}$ Analog to Digital Converter (ADC) for triggering purposes. Given that a large subset of MEG II detectors use SiPM technologies, the WaveDREAM was designed to provide the required bias voltage by means of an optional generator circuit, and contains a programmable amplifier up to gain 100 with tunable Pole-Zero compensation to operate in the high rate environment of MEG II. Thus, the 16 readout channels of the WaveDREAM can be directly connected to the sensors without any need of external equipment, providing therefore an extremely compact DAQ system, with a 3 rack-unit crate containing up to 256 channels.

The readout of WaveDREAMs within a crate is sequenced through a Data Concentration Board (DCB) over a standard Gigabit Ethernet link. That same board also provides access to the WaveDREAM for configuration purposes and distributes the clock, trigger and synchronisation signals within the crate.

Ethernet packets are collected, at a million packets per second speed, by a set of readout threads on the main DAQ computer and then, after event building and calibration, they are handed to the experiment MIDAS DAQ system [43].

Trigger inputs from the WaveDREAMs must be combined into complex trigger selections that are mandatory to reduce the $7 \times 10^{7} \mu^{+} / \mathrm{s}$ to a manageable rate (order of $10 \mathrm{~Hz}$ ). In particular the ADCs are used to estimate the energy deposit in the liquid xenon detector through a weighted sum algorithm similar to the one used in MEG [44]. The inclusion of a discriminator in the WaveDREAM opens the possibility to obtain a hit timestamp with a precision higher than in MEG by sampling the discriminated value several times within the ADC clock period of $80 \mathrm{MHz}$. Operating with the maximum oversampling by a factor 8 , the timestamp information available at trigger level is available with a $1.56 \mathrm{~ns}$ time bin. 
The combination of trigger information is performed through a three level tree by a custom made Field Programmable Gate Array board, named Trigger Concentrator Board (TCB) [45]. One of these boards is located in each crate containing WaveDREAMs, so as to forward the information out of the crate into a dedicated trigger crate where the last merging happens. Several complex trigger algorithms can be executed at the same time, also including pulse-shape based selections, such as to discriminate ${ }^{241} \mathrm{Am}$ induced alpha events from the gamma background [46].

\section{Results}

The MEG II experiment is currently under commissioning at the Paul Scherrer Institute. Since 2017 each detector underwent a set of "Pre-Engineering" runs by sharing a limited amount of readout channels; performances previously obtained on small scale prototypes were validated and the estimate of the experiment sensitivity was then confirmed to be $6 \times 10^{-14}$ at $90 \%$ confidence level [47]. In autumn 2021, the first engineering run with the full detectors will be completed with a preliminary physics dataset collected soon after.

In spring 2021, the complete TDAQ system was finally installed in the experimental area (Figure 6), opening further possibilities of detector performance studies, in particular for the positron spectrometer. This was indeed a significant milestone for the experiment, being crucial to start combining pieces of information of the different detectors toward the future physics run.
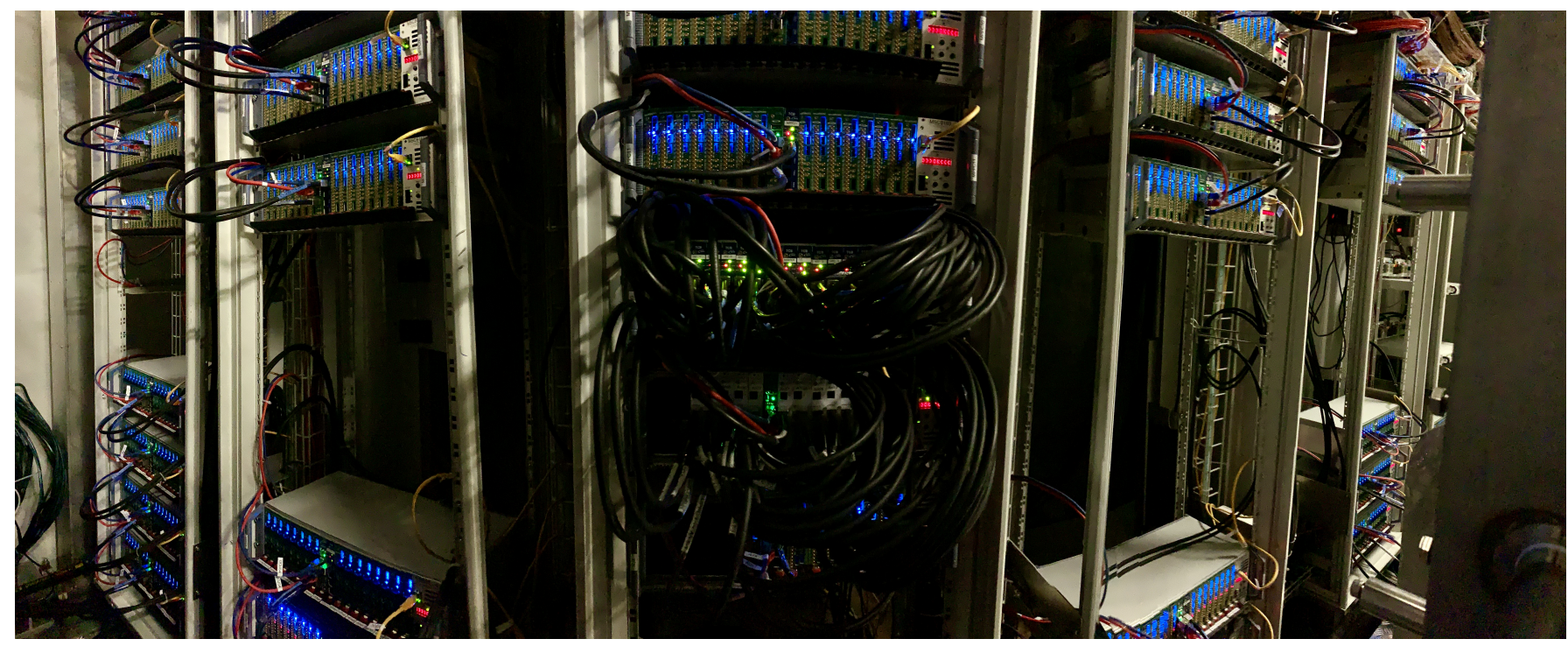

Figure 6. Panoramic picture of the TDAQ system completely installed in the experimental hall. Blue LEDs are from WaveDREAM cards, the different boards in the middle of each crate are DCBs (on the left) and TCBs (on the right). The two central crates with black cables are the ones used for trigger generation and distribution.

\subsection{Pre-Engineering Runs: Results \\ 3.1.1. pTC}

The pTC performance and stability were assessed in the 2017 pre-engineering run. The hit rate, radiation damage and resolution were studied with the $\mu^{+}$beam at the intensity designed for MEG II. The overall detector resolution is estimated to be 35 ps: this value is obtained by dividing the single hit resolution by the square root of the number of hits of a signal $\mathrm{e}^{+}$, which is 9 on average. The single counter resolution, measured in laboratory with a ${ }^{90} \mathrm{Sr}$ source, is on average $72 \mathrm{ps}$ and $81 \mathrm{ps}$ for $W=40 \mathrm{~mm}$ and $W=50 \mathrm{~mm}$ scintillators. The discrepancy between these values and the overall resolution measured in the experiment is due to the larger noise contribution observed in the MEG II environment.

The resolution is expected to deteriorate to $41 \mathrm{ps}$ at the end of the MEG II physics run because of radiation damage predicted over the full MEG II data taking period, but it still 
satisfies the measurement requirement. The deterioration originates from the increase of the dark current $I_{\text {dark }}$ in the SiPMs: irradiation tests performed in laboratory resulted in a linear increase of $\sigma_{t}^{2}$ with increasing $I_{\text {dark }}$ [28]. Such tests were carried out using a ${ }^{90}$ Sr source up to $\sim 1 \mathrm{kGy}$ and neutrons up to a $1 \mathrm{MeV}$ neutron equivalent flux of $\Phi_{\mathrm{eq}} \approx 5.5 \times 10^{9} \mathrm{~cm}^{-2}$. The damage level accumulated in the three years expected for the MEG II run is estimated to be $\Phi_{\mathrm{eq}} \approx 3 \times 10^{9} \mathrm{~cm}^{-2}$ : the resolution deterioration observed at this irradiation level is of $41 \%$, but this value decreases up to $13 \%$ using the pTC cooling system at $10{ }^{\circ} \mathrm{C}$, which is the temperature setting chosen for the pre-engineering runs and the physics run.

In the 2020 engineering run only the upstream module of the pTC was installed to be used as a trigger for the $\mathrm{CDCH}$. Because of the few available electronics channels only half of the module was read out with a scheme optimised to match the CDCH readout. Since the commissioning of the detector ended in 2017, the 2020 run was used to further test the pTC stability, both under muon beam and, for the first time, under the pion beam used in the CEX run for LXe calibration, with successful results.

\subsection{2. $\mathrm{CDCH}$}

The CDCH was transported to PSI in the Summer 2018 after the assembly in Pisa (Italy) for the commissioning phase and the full integration in the MEG II experimental apparatus [48]. The final wire stretching was set and the HV working point was reached. The CDCH was tested during the engineering runs 2018, 2019 and 2020 performing HV scans around the working point with different ionization sources. After a period of conditioning, the chamber operational stability was tested with $\mu^{+}$beam at different intensities up to the MEG II one. Michel positrons from muon decay were acquired with a pTC trigger. Anomalously high currents (up to $300 \mu \mathrm{A}$ ) were observed in some sectors. These were cured through a gas mixture optimization with the addition of up to $2 \% \mathrm{O}_{2}$, which was then gradually reduced to a lower content. The chamber was operated in stable conditions at the full MEG II beam intensity with the standard gas mixture + isopropyl alcohol $(1 \%)+\mathrm{O}_{2}(0.5 \%)$ for a period of about one week during the 2020 run. The gas gain was determined with cosmic rays in a clean environment, resulting to be $(4 \div 7) \times 10^{5}$, as expected and demonstrated to be sensitive to the single ionization cluster, thus allowing to explore cluster timing techniques [49]. The noise level in the experimental environment was thoroughly studied, and found to be higher than initially expected. Digital filters were developed to cope with it. FE electronics with three different amplification stages were tested to investigate the optimal electronics gain value. The configuration with the highest gain was found to have the best signal-to-noise ratio and it is currently used (bandwidth around $380 \mathrm{MHz}$ ). The impact of added $\mathrm{O}_{2}$ at concentrations at the $0.5 \%$ level or below on the $\mathrm{CDCH}$ performance is expected to be not significant. Since a limited number of DAQ channels were available during the engineering runs, an accurate measurement of the chamber performance with data was not possible. Nevertheless, this partial readout was crucial to refine and tune the diagnostic and reconstruction algorithms on real data. Full MC studies show momentum, angular and vertex resolutions of $100 \mathrm{keV} / \mathrm{c}, 6.7 \mathrm{mrad}$ and $1.7 / 0.8 \mathrm{~mm}$ (longitudinal/transverse) respectively, in agreement with the MEG II experimental requirements.

\subsubsection{LXe}

The liquid xenon detector was transported to the experimental area in the Summer of 2017 after the replacement of PMTs with MPPCs. The first objective was to measure the photosensor performance and its stability. Monitoring during the beamtime indicated that the MPPC PDE for VUV light decreased with the use of the beam and the MPPCs with less radiation exposure had higher PDE. A wavelength dependence in the PDE degradation was observed: the MPPC PDE for visible light (from blue LEDs) also decreased, but the degradation rate was lower than the one for VUV light by an order of magnitude. From these observations, this degradation is most likely due to surface damage induced by the radiation exposure to a high-intensity muon beam environment. VUV photons create 
electron-hole pairs in the shallow region of a MPPC, and hence the PDE for VUV light is sensitive to the surface state. Thermal annealing is known to be a solution to the surface damage [50] and was tested twice in 2019 and 2020. A higher bias voltage was applied to several MPPCs, and they were heated by Joule heat from the quench resistor. This recovered the PDE from the damaged level $(8-10 \%)$ to the original level $(18-20 \%)$. The overall impact of the radiation damage on the detector performances was studied on simulations. The degradation of the detector resolution was found to be limited because the position and energy resolution are not dominated by the photo-electron statistics. The time resolution is defined only by PMTs when the MPPC PDE is reduced. The collaboration is evaluating how to include the time needed for the annealing process in the run plan to minimize any eventual sensitivity loss by the reduced statistics.

The energy resolution around $52.8 \mathrm{MeV}$ was measured in two different ways. One method is based on the energy spectrum of the background gamma rays of ordinary muon decays, where the energy resolution is estimated by comparison with simulations. The other method uses two gamma rays with correlated energies and opening angles from the decay of neutral pions produced in CEX reactions. The energy resolution of both methods was measured to be $1.8 \%$, which was not as good as the resolution goal (1.0\%), but the energy resolution for shallow depths was improved as expected with respect to the MEG experiment. The reason for this discrepancy will be investigated in this year's Engineering run.

The position resolution was measured using a collimator with narrow slits. The width of peak structures in the observed position distribution was compared to the MC simulation. A clear improvement of the resolution in the shallow region was observed with respect to the MEG experiment and this is consistent with simulations. A position resolution of $2.5 \mathrm{~mm}$ is achieved on average, compared to a resolution of $\sim 5 \mathrm{~mm}$ in the MEG experiment. The position of the MPPCs is measured with an optical survey using a laser scanner and a movable low energy gamma-ray beam [51]. As a result, the MPPCs are aligned with respect to the COBRA magnet with a precision of $0.57 \mathrm{~mm}$ by the combination of the two measurements.

\subsection{Engineering Run 2021: Highlights}

Being the first run with the complete TDAQ system and all detectors in place, the 2021 Engineering run will see the development of full experiment reconstruction, including the commissioning of a preliminary $\mu^{+} \rightarrow \mathrm{e}^{+} \gamma$ trigger selection.

The pTC is ready for the MEG II physics run, and for the ongoing 2021 engineering run both US and DS modules are already installed. This is the first run with all the electronics channels connected simultaneously to both modules. Moreover, the $\mathrm{CDCH}$ is fully instrumented and read out, meaning that it will be possible to study the noise and temperature conditions in the final configuration. Figure 7 shows the relative rate seen by the counters in the 2020 and in the first weeks of the 2021 run. Further, it highlights the difference in the available readout channels. Both plots refer to the same nominal beam intensity, namely the one used in MEG $\left(3 \times 10^{7} \mu / \mathrm{s}\right)$.

During the 2021 engineering run the $\mathrm{CDCH}$ full electronics readout is tested for the first time. This period is crucial for a complete noise and signal check of all DAQ channels. The temperature in the endcap regions using all active electronics was already proven to be under control thanks to the cooling circuit, further improved this year during the run preparation. An extensive campaign for the identification and suppression of hardware noise sources is foreseen. The gas mixture is further optimized through dedicated conditioning periods with $\mu^{+}$beam. The reconstruction algorithms are tested and the $\mathrm{CDCH}$ performances evaluated on data to obtain the first experimental resolutions on the $\mathrm{e}^{+}$track parameters and efficiency. Figure 8 shows examples of occupancy plots from Michel $\mathrm{e}^{+}$events with a comparison between the 2020 (a) and 2021 (b) readouts. Most of the dead channels will be recovered during the next maintenance period. The four upper 
sectors are out of acceptance for signal $\mathrm{e}^{+}$and are usually not readout. Nevertheless they are equipped with FE electronics. The full $2 \pi$ readout is possible.
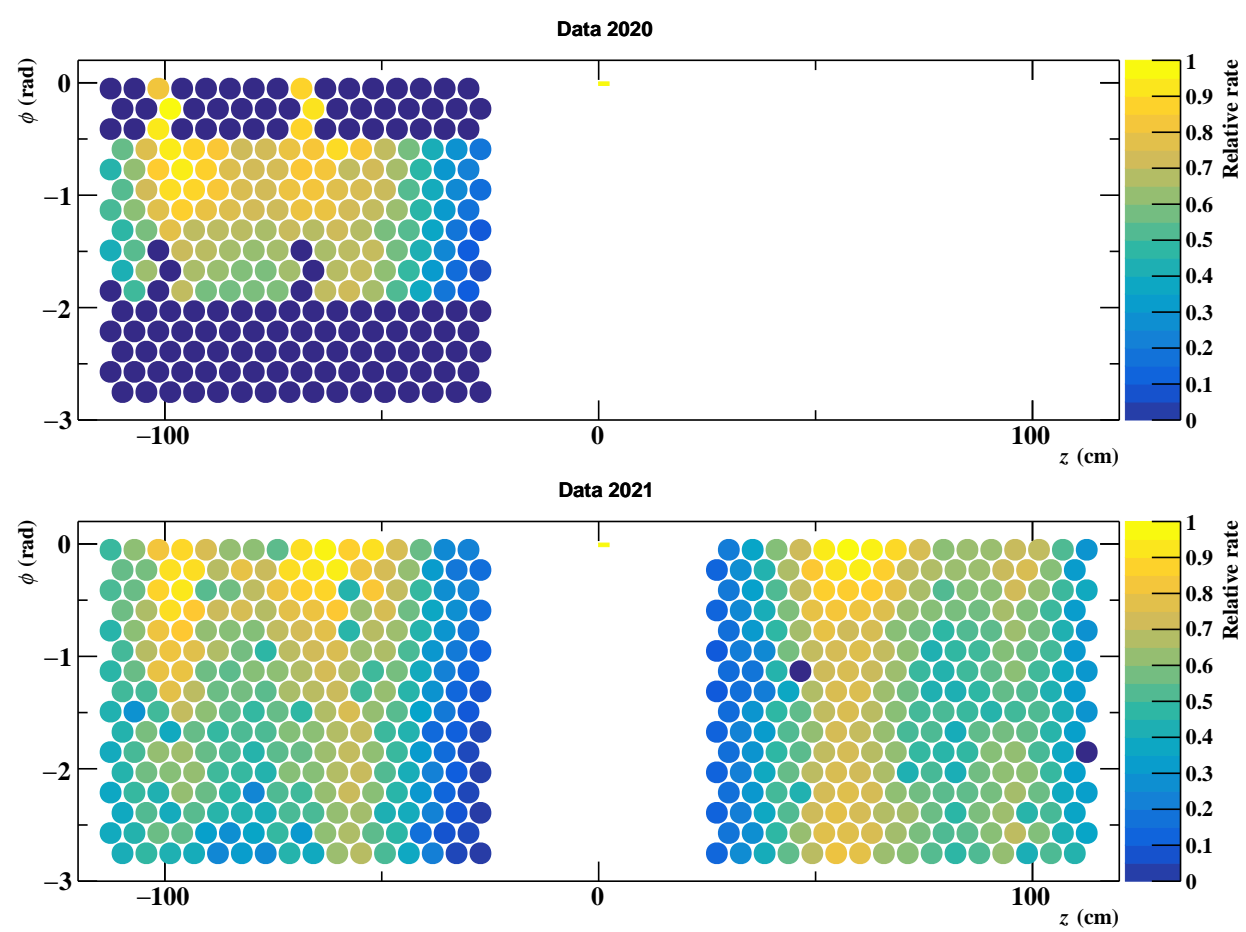

Figure 7. Relative hit rate of the pTC tiles in 2020 (top) and 2021 (bottom) runs.

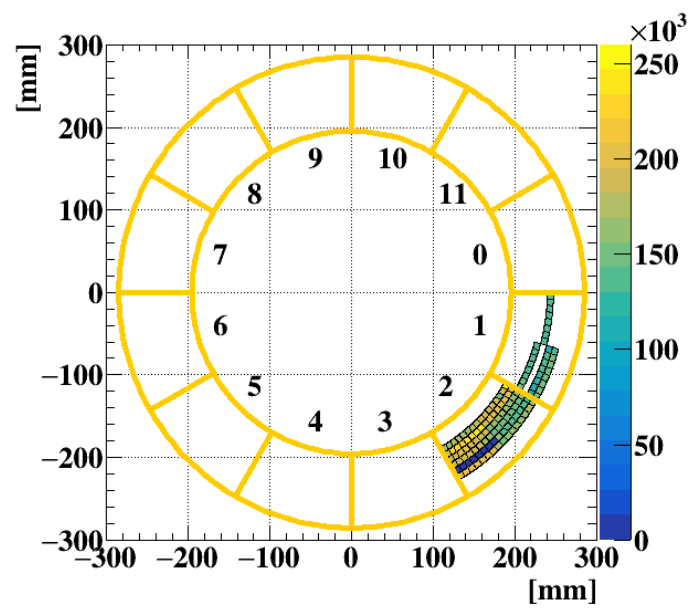

(a)

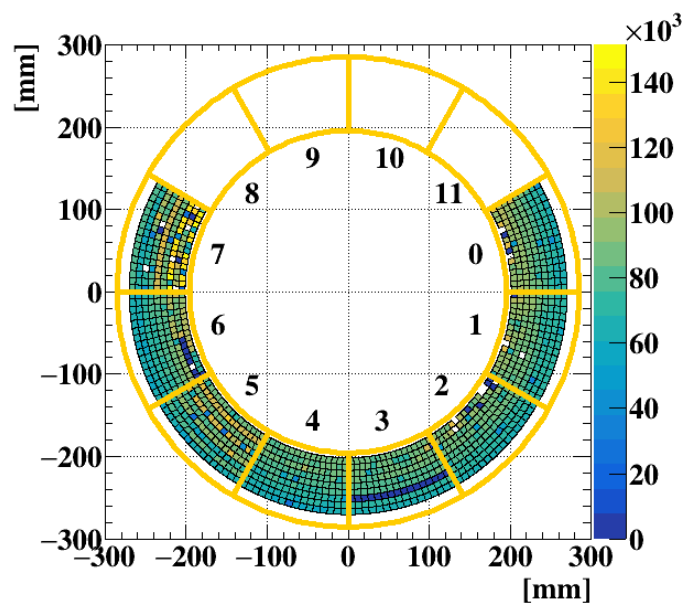

(b)

Figure 8. Occupancy plots from Michel $\mathrm{e}^{+}$events with a comparison between the 2020 (a) and 2021 (b) readouts.

As the liquid xenon gamma-ray detector is fully equipped with read out electronics, the detector performance over the entire detector can be assessed for the first time. The contribution of noise originating from the readout electronics to the energy resolution is equivalent to $0.04 \mathrm{MeV}$ and thus is negligible. Data acquisition of the gamma-ray spectrum from muon decays is already started together with constant calibrations to monitor the radiation damage. Since the energy reconstruction is based on the sum of the number of incident photons at each photosensor, the gradual development of radiation damage has to be constantly monitored by frequent calibration measurements. Figure 9 illustrates the event display of a gamma-ray pileup event. Thanks to the extended readout region, the position of pileup gamma-rays can be reconstructed from the light distribution on the 
entrance face. A measurement of the detection efficiency and the timing resolution for gamma-rays with the CEX calibration setup is planned at the end of the year.

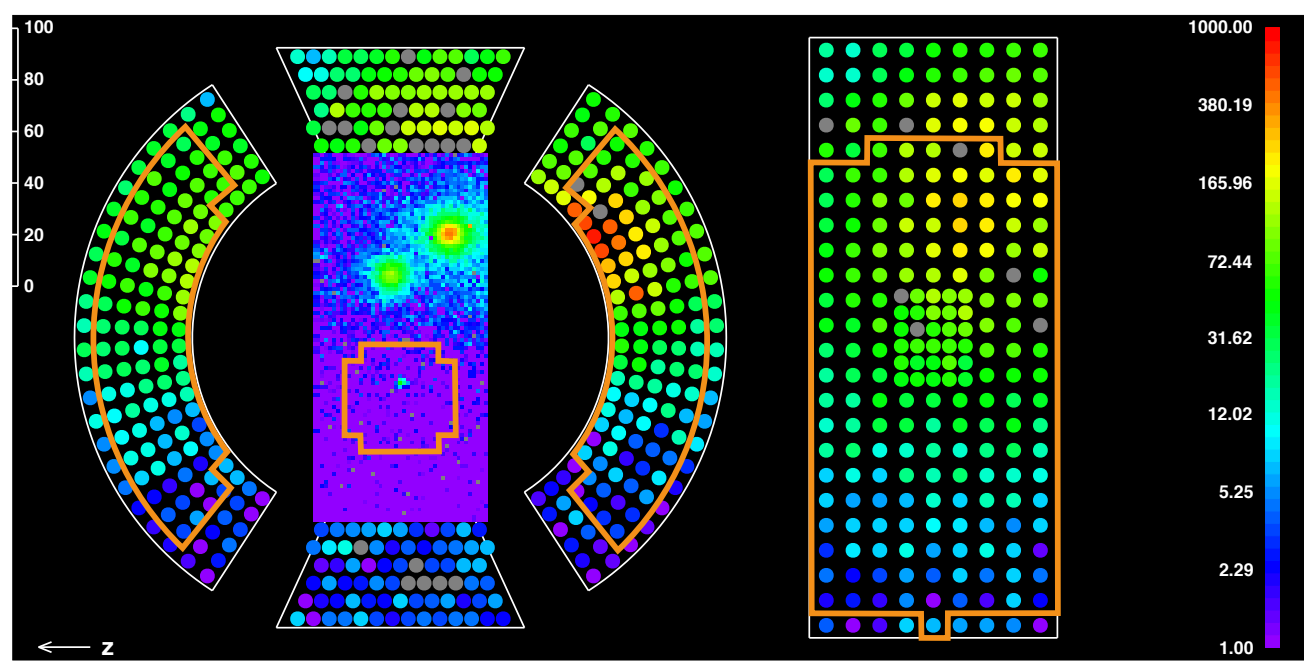

Figure 9. Gamma-ray event display with full readout. Readout region in 2020 is surrounded by orange lines.

\section{X(17) Boson Measurement}

During this year's run MEG II will additionally be able to perform the first test on a measurement concerning the so called X(17) boson. In 2016 a measurement at the Atomki laboratory (Debrecen, Hungary) revealed an anomaly in the form of an excess in the angular distribution of the Internal Pair Creation (IPC) in the nuclear reaction ${ }^{7} \mathrm{Li}\left(\mathrm{p}, \mathrm{e}^{+} \mathrm{e}^{-}\right)^{8} \mathrm{Be}$ [52]. This anomaly was confirmed by following measurements and most notably observed in the ${ }^{3} \mathrm{H}\left(\mathrm{p}, \mathrm{e}^{+} \mathrm{e}^{-}\right)^{4} \mathrm{He}$ reaction as well [53]. A possible interpretation of this observation is the production of a new physics boson mediator of a fifth fundamental force that describes the interaction between dark matter and ordinary matter [54]. This hypothetical boson is expected to have a mass of $17 \mathrm{MeV}$, hence the name $\mathrm{X}(17)$. Repeating this measurement with an independent experiment and a different angular coverage is crucial to confirm that the observed anomaly is not an artifact of the detector geometry, a possibility introduced in [55] to explain the excess within the SM.

The MEG II experiment has all the ingredients to repeat the Atomki measurement: the $\mathrm{CW}$ accelerator for the proton beam, the $\mathrm{CDCH}$ for $\mathrm{e}^{+} \mathrm{e}^{-}$measurement and the pTC as trigger. MC simulations showed that the MEG II spectrometer has better invariant mass resolution and angular acceptance with respect to the Atomki experiment. To maximize the resolution the $\mathrm{CW}$ target region was redesigned in order to minimize multiple scattering. The new setup consists of a $10 \mu \mathrm{m}$ thick $\mathrm{Li}_{2} \mathrm{O}$ layer on a $25 \mu \mathrm{m}$ thick $\mathrm{Cu}$ substrate, which is connected to the $\mathrm{CW}$ beamline by means of a $\mathrm{Cu}$ arm. Both structures are placed in a carbon fiber vacuum chamber. This configuration is incompatible with the LXe calibrations, but the configurations can be changed with some effort. For this reason the ${ }^{8}$ Be measurement will be completed at the beginning of 2022, after the 2021 engineering run. Nevertheless, during this year's run it will be possible to test the new target, support structure and vacuum chamber. In addition, some preliminary data will be taken with an intermediate setup compatible with MEG II calibrations, since it uses a smaller vacuum chamber that does not interfere with the CW insertion system.

\section{Conclusions}

MEG II is the upgrade of the MEG experiment, built to look for the CLFV decay $\mu^{+} \rightarrow \mathrm{e}^{+} \gamma$. The design of this upgrade makes it possible to achieve a sensitivity of $6 \times 10^{-14}$ in this search, an order of magnitude better than MEG. This is possible thanks to the increased granularity of the detectors, the larger acceptance and reduced material, in addition to better radiation hardness to cope with the high intensity $\mu^{+}$beam at PSI. 
The commissioning of the detectors that compose the experiment started in 2015 with the first pre-engineering run for the first estimate of the pTC time resolution and stability. The 2018 run was the first one with all detectors installed, allowing the first estimate of the their stability and performances, even though the number of readout channels was limited. These studies were carried on until 2020, and several problems arose during the years. Most notably the CDCH suffered of wire breaks and internal discharges under muon beam. Further, the LXe observed a decrease of the MPPC PDE at an unexpected rate. Solutions for these issues have been found, but a more detailed understanding of the long term behavior of these detectors is fundamental for the experiment, which is the goal of the 2021 run.

For the first time all the detectors are installed and fully connected to the readout, making the finalization of the performance assessment possible. Currently, the run is ongoing and the MPPC PDE and CDCH stability are being extensively tested, in parallel with the final trigger developments. The ultimate goal is to collect a first physics dataset by the end of the year, laying the foundation for the upcoming three years of MEG II physics runs.

Author Contributions: Investigation, M.C., M.F., S.K., M.M., R.O. and P.S. All authors have read and agreed to the published version of the manuscript.

Funding: This research was funded by DOE DEFG02-91ER40679 (USA), INFN, MIUR Montalcini D.M. 2014 n. 975 (Italy), JSPS Core-to-Core Program, A. Advanced Research Networks JPJSCCA20180004, JSPS KAKENHI Grant Numbers JP26000004, JP18K13557, JP19J13635, JP19J21535, JP19J21730, JP20H00154, JP21H00065 (Japan), the Russian Federation Ministry of Science and Higher Education (Russia), and Schweizerischer Nationalfonds (SNF) Grant 200020_172706 and Grant 206021_177038 (Switzerland).

Acknowledgments: The authors recognize and are thankful to the entire collaboration for the efforts in the commissioning of the MEG II experiment, as well as for the opportunity to present such preliminary results to the public. We are also grateful for the support and co-operation provided by PSI as the host laboratory and to the technical and engineering staff of our institutes.

Conflicts of Interest: The authors declare no conflict of interest.

\section{References}

1. Calibbi, L.; Signorelli, G. Charged Lepton Flavor Violation: An experimental and theoretical introduction. Riv. Nuovo Cimento 2018, 41, 71-174. [CrossRef]

2. Hincks, E.P.; Pontecorvo, B. On the absence of photons among the decay products of the 2.2 microsecond meson. Can. J. Res. 1950, 28a, 29-43. [CrossRef]

3. Fukuda, Y.; Hayakawa, T.; Ichihara, E.; Inoue, K.; Ishihara, K.; Ishino, H.; Itow, Y.; Kajita, T.; Kameda, J.; Kasuga, S.; et al. Evidence for Oscillation of Atmospheric Neutrinos. Phys. Rev. Lett. 1998, 81, 1562. [CrossRef]

4. Kuno, Y.; Okada, Y. Muon decay and physics beyond the standard model. Rev. Mod. Phys. 2001, 73, 151-202. [CrossRef]

5. Hernández-Tomé, G.; Castro, G.L.; Roig, P. Flavor violating leptonic decays of $\tau$ and $\mu$ leptons in the Standard Model with massive neutrinos. Eur. Phys. J. C 2019, 79, 84. [CrossRef]

6. Calibbi, L.; López-Ibáñez, M.L.; Melis, A.; Vives, O. Implications of the Muon g-2 result on the flavour structure of the lepton mass matrix. arXiv 2021, arXiv:2104.03296.

7. Abi, B.; Albahri, T.; Al-Kilani, S.; Allspach, D.; Alonzi, L.P.; Anastasi, A.; Anisenkov, A.; Azfar, F.; Badgley, K.; Baeßler, S.; et al. Measurement of the Positive Muon Anomalous Magnetic Moment to 0.46 ppm. Phys. Rev. Lett. 2021, 126, 141801. [CrossRef] [PubMed]

8. Baldini, A.M.; Bao, Y.; Baracchini, E.; Bemporad, C.; Berg, F.; Biasotti, M.; Boca, G.; Cascella, M.; Cattaneo, P.W.; Cavoto, G.; et al. Search for the lepton flavour violating decay $\mu^{+} \rightarrow \mathrm{e}^{+} \gamma$ with the full dataset of the MEG experiment. Eur. Phys. J. C 2016, 76, 434. [CrossRef]

9. Davidson, S. Completeness and complementarity for $\mu \rightarrow e \gamma, \mu \rightarrow e \bar{e} e$ and $\mu A \rightarrow e A$. J. High Energy Phys. 2021, $2021,1-36$.

10. Arndt, K.; Augustin, H.; Baesso, P.; Berger, N.; Berg, F.; Betancourt, C.; Bortoletto, D.; Bravar, A.; Briggl, K.; vom Bruch, D.; et al. Technical design of the phase I Mu3e experiment. Nucl. Instrum. Methods Phys. Res. Sect. A Accel. Spectrometers Detect. Assoc. Equip. 2021, 1014, 165679. [CrossRef]

11. Bartoszek, L.; Barnes, E.; Miller, J.P.; Mott, J.; Palladino, A.; Quirk, J.; Roberts, B.L.; Crnkovic, J.; Polychronakos, V.; Tishchenko, V.; et al. Mu2e Technical Design Report. arXiv 2014, arXiv:1501.05241.

12. Abramishvili, R.; Adamov, G.; Akhmetshin, R.R.; Allin, A.; Angélique, J.C.; Anishchik, V.; Aoki, M.; Aznabayev, D.; Bagaturia, I.; Ban, G.; et al. COMET Phase-I Technical Design Report. Prog. Theor. Exp. Phys. 2020, 2020, 033C01. [CrossRef] 
13. Nguyen, T.M. [DeeMe Collaboration]. Search for $\mu \rightarrow e$ conversion with DeeMe experiment at J-PARC MLF. PoS FPCP 2015, 248, 060 .

14. Adam, J.; Bai, X.; Baldini, A.M.; Baracchini, E.; Bemporad, C.; Boca, G.; Cattaneo, P.W.; Cavoto, G.; Cei, F.; Cerri, C.; et al. The MEG detector for $\mu^{+} \rightarrow \mathrm{e}^{+} \gamma$ decay search. Eur. Phys. J. C 2013, 73, 2365. [CrossRef]

15. Baldini, A.M.; Bao, Y.; Baracchini, E.; Bemporad, C.; Berg, F.; Biasotti, M.; Boca, G.; Cattaneo, P.W.; Cavoto, G.; Cei, F.; et al. Measurement of the radiative decay of polarized muons in the MEG experiment. Eur. Phys. J. C 2016, 76, 108. [CrossRef]

16. Baldini, A.M.; Baracchini, E.; Bemporad, C.; Berg, F.; Biasotti, M.; Boca, G.; Cattaneo, P.W.; Cavoto, G.; Cei, F.; Chiappini, M.; et al. The design of the MEG II experiment. Eur. Phys. J. C 2018, 78, 380. [CrossRef]

17. PSI. The Swiss Research Infrastructure for Particle Physics CHRISP. Available online: https://www.psi.ch/en/media/chrispoverview (accessed on 25 November 2021).

18. Baldini, A.M.; Bao, Y.; Baracchini, E.; Bemporad, C.; Berg, F.; Biasotti, M.; Boca, G.; Cattaneo, P.W.; Cavoto, G.; Cei, F.; et al. Muon polarization in the MEG experiment: Predictions and measurements. Eur. Phys. J. C 2016, 76, 223. [CrossRef]

19. Papa, A.; Barchetti, F.; Gray, F.; Ripiccini, E.; Rutar, G. A multi-purposed detector with silicon photomultiplier readout of scintillating fibers. Nucl. Instr. Meth. A 2015, 787, 130-133. [CrossRef]

20. Papa, A.; Rutar, G.; Barchetti, F.; Hildebrandt, M.; Kettle, P.R. A fast and quasi non-invasive muon beam monitor working at the intensity frontier. Nucl. Instr. Meth. A 2019, 936, 634-635. [CrossRef]

21. Cavoto, G.; Chiarello, G.; Hildebrandt, M.; Hofer, A.; Ieki, K.; Meucci, M.; Milana, S.; Pettinacci, V.; Renga, F.; Voena, C. A photogrammetric method for target monitoring inside the MEG II detector. Rev. Sci. Instrum. 2021, 92, 043707. [CrossRef] [PubMed]

22. Palo, D.; Hildebrandt, M.; Hofer, A.; Kyle, W.; Lad, D.; Libeiro, T.; Molzon, W. Precise Photographic Monitoring of MEG II Thin-film Muon Stopping Target Position and Shape. Nucl. Instrum. Methods A 2019, 944, 162511. [CrossRef]

23. Ootani, W.; Odashima, W.; Kimura, S.; Kobayashi, T.; Makida, Y.; Mitsuhashi, T.; Mizumaki, S.; Ruber, R.; Yamamoto, A. Development of a thin-wall superconducting magnet for the positron spectrometer in the MEG experiment. IEEE Trans. Appl. Supercond. 2004, 14, 568-571. [CrossRef]

24. Nishimura, M.; Berg, F.; Biasotti, M.; Boca, G.; Cattaneo, P.W.; De Gerone, M.; De Bari, A.; Francesconi, M.; Galli, L.; Gatti, F.; et al Full system of positron timing counter in MEG II having time resolution below 40 ps with fast plastic scintillator readout by SiPMs. Nucl. Instrum. Methods A 2020, 958, 162785. [CrossRef]

25. Saint-Gobain Ceramics \& Plastics, Inc. BC-418, BC-420, BC-422 Premium Plastic Scintillators. Available online: http:/ www crystals.saint-gobain.com/sites/imdf.crystals.com/files/documents/sgc-bc418-420-422-data-sheet_69699.pdf (accessed on 25 November 2021).

26. 3M. ESR Reflector Datasheet. Available online: https://www.3m.com/3M/en_US/p/d/eebgdar000006/ (accessed on 25 November 2021).

27. Boca, G.; Cattaneo, P.W.; De Gerone, M.; Francesconi, M.; Galli, L.; Gatti, F.; Koga, J.; Nakao, M.; Nishimura, M.; Ootani, W.; et al. The laser-based time calibration system for the MEG II pixelated Timing Counter. Nucl. Instrum. Methods A 2019, $947,162672$. [CrossRef]

28. Boca, G.; Cattaneo, P.W.; De Gerone, M.; Gatti, F.; Nakao, M.; Nishimura, M.; Ootani, W.; Rossella, M.; Uchiyama, Y.; Usami, M.; et al. Timing resolution of a plastic scintillator counter read out by radiation damaged SiPMs connected in series. Nucl. Instrum. Methods A 2021, 999, 165173. [CrossRef]

29. Chiappini, M.; Baldini, A.M.; Cavoto, G.; Cei, F.; Chiarello, G.; Francesconi, M.; Galli, L.; Grancagnolo, F.; Grassi, M.; Hildebrandt, M.; et al. The new drift chamber of the MEG II experiment. Nucl. Instrum. Methods Phys. Res. Sect. A Accel. Spectrometers Detect. Assoc. Equip. 2019, 936, 501-502. [CrossRef]

30. Baldini, A.M.; Cavoto, G.; Cei, F.; Chiappini, M.; Chiarello, G.; Corvaglia, A.; Francesconi, M.; Galli, L.; Grancagnolo, F.; Grassi, M.; et al. The ultra light Drift Chamber of the MEG II experiment. Nucl. Instrum. Methods Phys. Res. Sect. A Accel. Spectrometers Detect. Assoc. Equip. 2020, 958, 162152. [CrossRef]

31. Chiarello, G.; Chiri, C.; Corvaglia, A.; Grancagnolo, F.; Miccoli, A.; Panareo, M.; Pinto, C.; Spedicato, M.; Tassielli, G.F. The construction technique of the high granularity and high transparency drift chamber of MEG II. J. Instrum. 2017, 12, C07022. [CrossRef]

32. Baldini, A.M.; Baracchini, E.; Cavoto, G.; Cei, F.; Chiappini, M.; Chiarello, G.; Chiri, C.; Francesconi, M.; Galli, L.; Grancagnolo, F.; et al. Gas Distribution and Monitoring for the Drift Chamber of the MEG-II Experiment. J. Instrum. 2018, 13, P06018. [CrossRef]

33. Baldini, A.M.; Baracchini, E.; Cavoto, G.; Cascella, M.; Cei, F.; Chiappini, M.; Chiarello, G. Chiri, C.; Dussoni, S.; Galli, L.; et al. Single-hit resolution measurement with MEG II drift chamber prototypes. J. Instrum. 2016, 11, P07011. [CrossRef]

34. Venturini, M.; Baldini, A.M.; Baracchini, E.; Cei, F.; Dussoni, S.; Galli, L.; Grassi, M.; Nicolò, D.; Signorelli, G.; Tenchini, F.; et al. Ageing tests for the MEG II drift chamber. Nucl. Instrum. Methods Phys. Res. Sect. A Accel. Spectrometers Detect. Assoc. Equip. 2016, 824, 592-594. [CrossRef]

35. Chiarello, G.; Chiri, C.; Corvaglia, A.; Grancagnolo, F.; Panareo, M.; Pepino, A.; Pinto, C.; Tassielli, G. A high performance Front End Electronics for drift chamber readout in MEG experiment upgrade. Nucl. Instrum. Methods A 2016, 824, 336-339. [CrossRef]

36. Baldini, A.M.; Cavoto, G.; Cei, F.; Chiappini, M.; Chiarello, G.; Chiri, C.; Cocciolo, G.; Corvaglia, A.; Cuna, F.; Francesconi, M.; et al. Detailed analysis of chemical corrosion of ultra-thin wires used in drift chamber detectors. arXiv 2021, arXiv:2108.13948. 
37. Ieki, K.; Iwamoto, T.; Kaneko, D.; Kobayashi, S.; Matsuzawa, N.; Mori, T.; Ogawa, S.; Onda, R.; Ootani, W.; Sawada, R.; et al. Large-area MPPC with enhanced VUV sensitivity for liquid xenon scintillation detector. Nucl. Instrum. Methods A 2019, 925, 148-155. [CrossRef]

38. Baldini, A.M.; Bemporad, C.; Cei, F.; Dussoni, S.; Gatti, F.; Grassi, M.; Haruyama, T.; Hisamatsu, Y.; Iwamoto, T.; Mihara, S.; et al. A radioactive point-source lattice for calibrating and monitoring the liquid xenon calorimeter of the MEG experiment. Nucl. Instrum. Methods A 2006, 565, 589-598. [CrossRef]

39. Adam, J.; Bai, X.; Baldini, A.; Baracchini, E.; Bemporad, C.; Boca, G.; Cattaneo, P.W.; Cavoto, G.; Cei, F.; Cerri, C.; et al. Calibration and monitoring of the MEG experiment by a proton beam from a Cockcroft-Walton accelerator. Nucl. Instrum. Methods A 2011, 641, 19-32. [CrossRef]

40. Oya, A.; Ieki, K.; Ochi, A.; Onda, R.; Ootani, W.; Yamamoto, K. Development of high-rate capable and ultra-low mass Resistive Plate Chamber with Diamond-Like Carbon. arXiv 2021, arXiv:2109.13525.

41. Ritt, S.; Dinapoli, R.; Hartmann, U. Application of the DRS chip for fast waveform digitizing. Nucl. Instrum. Methods A 2010, 623, 486-488. [CrossRef]

42. Galli, L.; Baldini, A.M.; Cei, F.; Chiappini, M.; Francesconi, M.; Grassi, M.; Hartmann, U.; Meucci, M.; Morsani, F.; Nicolò, D.; et al WaveDAQ: An highly integrated trigger and data acquisition system. Nucl. Instrum. Methods A 2019, 936, 399-400. [CrossRef]

43. Ritt, S. MIDAS: Midas.psi.ch. Available online: http:// midas.psi.ch (accessed on 25 November 2021).

44. Galli, L.; Baldini, A.M.; Cattaneo, P.W.; Cei, F.; De Gerone, M.; Dussoni, S.; Gatti, F.; Grassi, M.; Morsani, F.; Nicolò, D.; et al. Operation and performance of the trigger system of the MEG experiment. J. Instrum. 2014, 9, P04022. [CrossRef]

45. Francesconi, M.; Baldini, A.M.; Cei, F.; Chiappini, M.; Galli, L.; Grassi, M.; Hartmann, U.; Morsani, F.; Nicolò, D.; Papa, A.; et al. Low latency serial communication for MEG II trigger system. Nucl. Instrum. Methods Phys. Res. Sect. A Accel. Spectrometers Detect. Assoc. Equip. 2019, 936, 331-332. [CrossRef]

46. Nicolò, D.; Baldini, A.M.; Bemporad, C.; Cei, F.; Chiappini, M.; Francesconi, M.; Galli, L.; Grassi, M.; Iwamoto, T.; Morsani, F.; et al. Real-time particle identification in liquid xenon. IEEE Trans. Nucl. Sci. 2021, 68, 2630-2636. [CrossRef]

47. Baldini, A.M.; Bao, Y.; Baracchini, E.; Bemporad, C.; Berg, F.; Biasotti, M.; Boca, G.; Cascella, M.; Cattaneo, P.W.; Cavoto, G.; et al. The Search for $\mu \rightarrow$ e $\gamma$ with 10-14 Sensitivity: The Upgrade of the MEG Experiment. Symmetry 2021, 13, 1591. [CrossRef]

48. Chiappini, M.; Baldini, A.M.; Cavoto, G.; Cei, F.; Chiarello, G.; Corvaglia, A.; Francesconi, M.; Galli, L.; Grancagnolo, F.; Grassi, M.; et al. Commissioning of the MEG II tracker system. J. Instrum. 2020, 15, C06056. [CrossRef]

49. Cascella, M.; Grancagnolo, F.; Tassielli, G. Cluster Counting/Timing Techniques for Drift Chambers. Nucl. Phys. B (Proc. Suppl.) 2014, 248-250, 127-130. [CrossRef]

50. Li, A.N.F. CCD Image Sensors in Deep-Ultraviolet; Springer: Berlin/Heidelberg, Germany, 2005. [CrossRef]

51. Kobayashi, S.; Francesconi, M.; Galli, L.; Ieki, K.; Iwamoto, T.; Libeiro, T.; Matsuzawa, N.; Molzon, W.; Mori, T.; Nakao, M.; et al. Precise measurement of 3D-position of SiPMs in the liquid xenon gamma-ray detector for the MEGII experiment. Nucl. Instrum. Methods Phys. Res. Sect. A Accel. Spectrometers Detect. Assoc. Equip. 2019, 936, 189-191. [CrossRef]

52. Krasznahorkay, A.J.; Csatlós, M.; Csige, L.; Gácsi, Z.; Gulyás, J.; Hunyadi, M.; Kuti, I.; Nyakó, B.M.; Stuhl, L.; Timár, J.; et al. Observation of anomalous internal pair creation in ${ }^{8}$ Be: A possible indication of a light, neutral boson. Phys. Rev. Lett. 2016, 116, 042501. [CrossRef]

53. Krasznahorkay, A.J.; Csatlós, M.; Csige, L.; Gulyás, J.; Koszta, M.; Szihalmi, B.; Timár, J.; Firak, D.S.; Nagy, Á.; Sas, N.J.; et al. A new anomaly observed in 4 He supporting the existence of the hypothetical X17 particle. J. Phys. Conf. Ser. 2020, $1643,012001$. [CrossRef]

54. Feng, J.L.; Fornal, B.; Galon, I.; Gardner, S.; Smolinsky, J.; Tait, T.M.P.; Tanedo, P. Protophobic Fifth-Force Interpretation of the Observed Anomaly in ${ }^{8}$ Be Nuclear Transitions. Phys. Rev. Lett. 2016, 117, 071803. [CrossRef]

55. Aleksejevs, A.; Barkanova, S.; Kolomensky, Y.G.; Sheff, B. A Standard Model explanation for the "ATOMKI anomaly". arXiv 2021, arXiv:2102.01127. 